\title{
Val de Fensch : résilience, transformations et anticipations d'un territoire encore industriel
}

Val de Fensch: resilience, transformations and expectations of a still industrial territory

Val de Fensch : Widerstandsfähigkeit, Veränderungen und Erwartungen eines noch Industriegebiet.

\section{Eric Marochini}

\section{OpenEdition Journals}

Édition électronique

URL : http://journals.openedition.org/rge/9286

DOI : $10.4000 /$ rge.9286

ISSN : 2108-6478

Éditeur

Association des géographes de l'Est

\section{Référence électronique}

Eric Marochini, «Val de Fensch : résilience, transformations et anticipations d'un territoire encore industriel », Revue Géographique de l'Est [En ligne], vol. 58/3-4 | 2018, mis en ligne le 21 juillet 2020, consulté le 08 septembre 2020. URL : http://journals.openedition.org/rge/9286 ; DOI : https://doi.org/ $10.4000 /$ rge. 9286

Ce document a été généré automatiquement le 8 septembre 2020

Tous droits réservés 


\title{
Val de Fensch : résilience, transformations et anticipations d'un territoire encore industriel
}

\author{
Val de Fensch : resilience, transformations and expectations of a still industrial \\ territory \\ Val de Fensch: Widerstandsfähigkeit, Veränderungen und Erwartungen eines \\ noch Industriegebiet.
}

Eric Marochini

\section{Introduction}

1 Dans les paysages, les géohistoriens sont à la recherche de traces (Veschambre, 2008), « ils aiment plonger dans les empilements du passé, dans les interstices de l'histoire pour en révéler des legs, des héritages » (Husson, 2016), lesquelles peuvent être tout à la fois des avantages ou des inconvénients pour aménager les territoires du présent et préparer ceux de l'avenir. Dans les territoires et les paysages industriels, l'histoire se lit souvent plus facilement tant les signes du passé jalonnent encore les espaces, qu'il s'agisse de friches d'usines, de cités d'habitation ou d'infrastructures. Dans ces paysages, et en particulier dans ceux qui font l'objet de cette publication, « les grandes usines sidérurgiques ont bouleversé violemment les paysages traditionnels » (Crouzet, 1997).

2 L'actuel territoire de la Communauté d'agglomération du Val de Fensch , ses 10 communes membres ${ }^{1}$, correspond peu ou prou à l'espace géographique couvert par le bassin versant de la Fensch, modeste rivière dont personne ne parlerait aujourd'hui si la famille De Wendel (Marseille, 2005), n'était pas venue s'installer sur ses rives, à Hayange, il y a maintenant un peu plus de 310 ans. La sidérurgie, de l'extraction du minerai de fer à la production de fonte ou d'acier, a tout organisé dans cet espace relativement restreint pour ses besoins et ceux des populations qu'elle a employées. 
Comme l'ont si justement dépeint Anne et Denis Mathis en 2017, le bassin industriel de la vallée de la Fensch "est à l'origine une juxtaposition de villes-usines dont la concentration entraîne un changement d'échelle systémique : de la ville-usine à la vallée industrielle ».

3 Cet article propose le regard d'un géographe-aménageur et n'a pas de prétention méthodologique sur le fond. Il vise humblement à montrer la résilience comprise " comme la capacité d'un territoire à être en mouvement, à anticiper des évolutions rapides, à s'en relever » (Villar et David, 2014) d'une vallée industrielle lorraine avec ses singularités, ses originalités dans un contexte géopolitique renouvelé, notamment par les questions frontalières. Il cherche aussi à expliquer en quoi cette vallée est devenue une terre d'expérimentations de la transformation des espaces mono-industriels vers une forme de diversification, tout en maintenant ses activités sidérurgiques à un haut niveau de technicité, de modernité alors que depuis 2012 plus une tonne d'acier n'y est produite.

4 Après avoir exposé la genèse de cette vallée autrefois rurale et rapidement devenue la " vallée usinière " si bien dépeinte par A. Printz (1966) et son fils M. Printz très récemment (2017), puis analyser les permanences et nouvelles influences territoriales qui innervent ce territoire dont la géographie urbaine réorganise ses inclinations politiques (Lévy et al., 2018), cet article exposera des exemples précis de requalifications de friches d'usines et d'actions plus globales portées par la Communauté d'Agglomération du Val de Fensch, pour rendre son territoire plus attractif malgré la conjugaison de nombreux handicaps et pour donner plus de lisibilité à son patrimoine industriel en tout point remarquable.

\section{D'une organisation territoriale industrialo- paternaliste à un patchwork urbain désorganisé}

5 Comme les autres vallées sidérurgiques de la Chiers, de l'Alzette et de l'Orne qui entaillent les côtes de Moselle, le paysage et l'organisation sociale de la vallée de la Fensch, jusqu'alors voués à une polyculture bien médiocre, ont été totalement bouleversés par les activités minières et sidérurgiques. Au milieu du XIX ${ }^{\text {ème }}$ siècle, malgré l'installation précoce de la famille de Wendel sur le site de la Rotonde à Hayange dès 1704 , à peine 8.000 habitants y résidaient.

6 En l'espace de quelques décennies, et tout particulièrement à partir de 1880 et la mise au point du célèbre procédé de traitement des minerais de fer phosphoreux de ThomasGilchrist, « elle va faire le plein en bâtiments, en cités et en hommes souvent d'origines étrangères (polonais, allemands, italiens, algériens, marocains...) » (Gehring et SaintDizier, 1983). Le processus d'occupation de l'espace a été quasiment le même dans toutes ces "vallées usinières " (Printz, 1966). Les maîtres des forges y avaient commencé par acquérir ou y construire des moulins, des forges, des mines puis des installations plus intégrées, plus consommatrices d'espaces et d'hommes : des hautsfourneaux, des aciéries, des laminoirs (Kaercher-Régnery, 1990). En plus d'une occupation industrielle de l'espace et tout particulièrement des fonds de vallée, il s'est agi de fixer les populations venues travailler dans les mines et les installations sidérurgiques. Les industriels, profitant de leur maîtrise foncière et quasiment politique de l'espace ${ }^{2}$ ont dès lors construit des cités, des espaces de vie sociale et en cela ont cherché à exercer une forme de domination dans tous les domaines et tout au 
long de la vie des ouvriers. De nombreux auteurs se sont penchés sur ce sujet du paternalisme inspiré des travaux sur la réforme sociale de Frédéric Le Play (Sihol, 2007), dont François de Wendel (Jeanneney, 2019) fut d'ailleurs un lecteur.

7 À partir du début de $\mathrm{XX}^{\text {ème }}$ siècle, la vallée se remplit très rapidement d'usines toujours plus dévoreuses d'espace de l'amont vers l'aval de la rivière Fensch, se déclinant en trois grandes entités avec des fonctionnements propres. Ils ont cependant en commun qu'ils s'affranchissent des limites communales. En cela, le modèle industriel fenschois d'occupation de l'espace ne coïncide pas avec celui des villes-usines planifiées traditionnelles très étudiées et pour certaines d'entre elles classées au patrimoine mondial de l'UNESCO (Crespi d'Adda en Italie, New Lanark en Ecosse...).

Dans la partie haute de la vallée, assise sur les bans communaux de Algrange, Nilvange, Knutange et Fontoy ${ }^{3}$, ce sont les installations de la Société Métallurgique de Knutange qui dominaient (hauts-fourneaux, aciérie, laminoirs). Depuis la partie basse de Nilvange jusqu'à Florange et la confluence de la rivière Fensch avec la Moselle, s'étirait ensuite une usine intégrée sur près de 12 kilomètres, propriété de la famille De Wendel. Celle-ci comprenait alors les sites de Hayange Saint-Jacques (site de British Steel aujourd'hui), ceux de Patural toujours à Hayange, de la Fenderie 4 à Serémange-Erzange, puis toutes les usines dites de laminage à froid et de revêtement des aciers à Florange. Le troisième ensemble était celui de Lorfonte, construit en 1890 à Uckange par les frères Stumm, industriels sarrois, où se sont dressaient jusqu'à 6 hauts-fourneaux produisant de la fonte de moulage spécifique à ce site.

9 La croissance industrielle inédite et rapide s'est accompagnée d'un développement urbain tout aussi exceptionnel. La planification de cette urbanisation rapide ne s'organisait pas à l'échelle de la commune mais bien à celle de l'usine sous l'influence d'un paternalisme patronal (SMK, De Wendel, Stumm) qui intervient alors très directement dans la "fabrique urbaine ». La construction des villes dans cette vallée s'est donc faite par touches successives à la façon d'un tableau pointilliste par secteurs autour des usines ou des mines nouvelles. Il en ressort une impression assez étrange d'architecture urbaine géométrique mais sectorisée, sans cohérence d'ensemble, ressemblant le plus souvent à un entrelacs assez confus. 
Figure 1 - Le Val de Fensch : un territoire et une démographie hyper-réactifs aux soubresauts économiques de l'échelle monde.

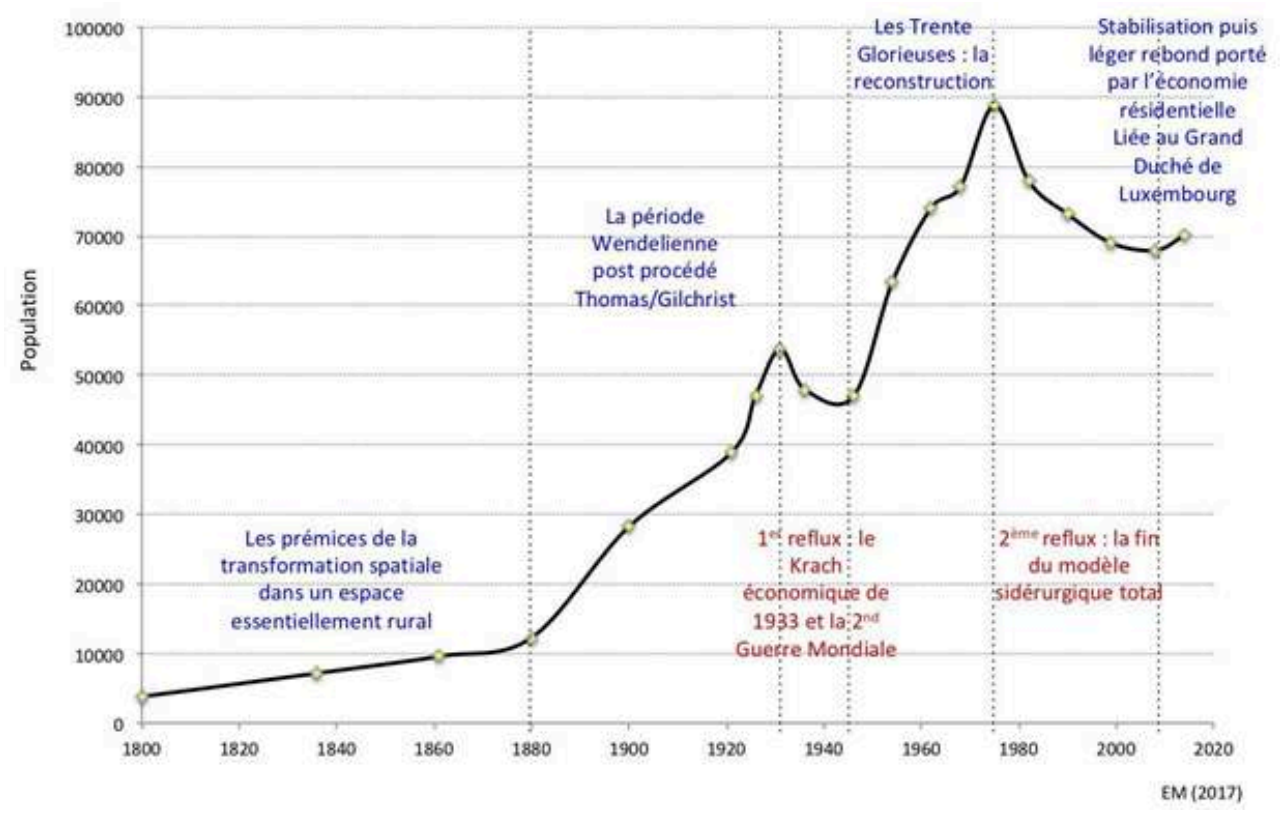

10 À Algrange par exemple, c'est autour des carreaux miniers dans la partie nord du finage et à proximité de l'usine SMK dite de la Paix dans sa partie sud que se fait le développement urbain. À Hayange, c'est d'abord autour des usines et dans des cités plus excentrées ensuite, au Konacker (presque ville nouvelle) ou encore à Saint-Nicolasen-Forêt, dernière ville française créée ex-nihilo puis rattachée à Hayange où avait été organisée une véritable ségrégation spatiale selon le niveau hiérarchique d'appartenance dans l'entreprise. La ville de Fameck, regroupement de hameaux très agricoles (Morlange, Rémelange, Budange, Edange), échappe au modèle de développement initial constaté dans les autres communes car plus éloignée des sites de production et disposant de terrains favorables à un développement horizontal. Avec la constitution du groupe SOLLAC (SOciété Lorraine de LAminage Continu) en 1948, elle connaît toutefois également une progression très spectaculaire mais plus tardive, elle suit le modèle de développement des « villes champignons ». Comme l'écrivent Anne et Denis Mathis en 2017 "le développement des usines de Florange et d'Ebange en aval de la vallée de la Fensch repousse les logements vers la périphérie. Ainsi dans les années 1960, les cités Oury sont construites à cheval sur les territoires de Florange et de Fameck accentuant encore l'éclatement communal fameckois ». Cette commune, comme Uckange et Florange, compte tenu de ses réserves foncières plus importantes, va connaître un développement nouveau entre 1950 et 1970 par la mise en chantier de vastes quartiers de logements dans ce que l'on appelait alors des Zones à Urbaniser en Priorité (ZUP).

11 La vallée prend peu à peu son allure caractéristique. Dans sa partie amont «l'usine en fond de vallée domine de ses blocs et de ses hautes cheminées des cités qui épousent les moindres replis de la vallée et ne laissent entre elles qu'un espace très chiche où se glissent la route et la voie ferrée " (Gehring, 1973). Dans sa partie avale, plus ouverte sur la vallée de la Moselle, le développement urbain s'est réalisé de manière plus mitée et plus tardive, l'usine est moins visible dans le paysage. Mais partout ailleurs, les espaces agricoles ont décru et les seules respirations vertes sont les forêts qui dominent les versants et une partie du plateau, dans la trilogie traditionnelle des paysages de cuesta. 
12 Au paroxysme de l'influence de l'industrie sur l'occupation de l'espace, par les usines, par l'habitat ouvrier, par les infrastructures logistiques, c'est-à-dire au début des années 1970 du siècle dernier, la population des 10 villes actuelles de la Communauté d'agglomération du Val de Fensch atteint elle-aussi son optimum, en l'occurrence un peu moins de 90.000 habitants (figure 1). Dès lors, avec les restructurations minières et sidérurgiques liées à une baisse massive des besoins, à la concurrence structurelle de la sidérurgie sur l'eau ${ }^{5}$, à la faible compétitivité du minerai de fer lorrain, et malgré tous les plans soutenus par l'Etat, commence un cycle de désindustrialisation des espaces assez singulier, différent de celui constaté ailleurs dans le bassin ferrifère lorrain. La partie amont de la vallée est la plus tôt concernée, avant même le premier choc pétrolier. Dès le milieu des années 1980, il n'y subsiste plus aucune industrie ou mine. L'usine SMK ferme ses portes en 1983 à Knutange, tout comme la dernière mine à Algrange (Mine "La Paix ») d'où est extraite l'ultime tonne de minerai en août de la même année ${ }^{6}$ (Figure 4).

Alors que les vallées de l'Orne et de la Chiers connaissent elles-aussi des fermetures brutales des usines et des mines, avec leur lot de drames sociaux, de régressions démographiques et urbaines, les parties centrale et avale de la vallée de la Fensch semblent un temps préservées du fait de nouveaux investissements à Florange en 1984 avec l'implantation par la SOLLAC d'une nouvelle usine dite "Sainte-Agathe " spécialisée dans le laminage à froid pour l'automobile, et par une réorganisation de la production de fonte et d'acier autour du site de Patural à Hayange, au détriment du site de « Saint Jacques » réservé au laminage des produits longs.

La seconde phase de recul industriel dans la vallée intervient en 1991, par l'aval, avec la fermeture très médiatisée de l'usine Lorfonte de Uckange ${ }^{7}$. C'est un nouveau traumatisme en particulier pour la commune de Uckange qui représentait parfaitement le modèle de la ville-usine. Après la dernière coulée du U1, le 18 décembre 1991, il n'y aura plus aucune activité sidérurgique sur le ban communal de Uckange. Dès lors, seuls deux hauts-fourneaux vont continuer à produire de la fonte dans la vallée de la Fensch, à Hayange sur le site de Patural, en amont d'une usine intégrée gigantesque traversant les communes de Hayange, Serémange-Erzange et Florange.

21 ans après la fermeture du U4, en 2012, ce sera au tour de Hayange et du site historique de Patural de connaître un sort identique. Il y eut jusqu'à 26 hautsfourneaux dans les 10 communes de l'actuel périmètre de la Communauté d'Agglomération du Val de Fensch (Printz et Schmidt, 2012), plus aucun ne fume aujourd'hui et seuls trois sont encore debout. À Uckange, il est devenu le totem d'un territoire industriel, un repère dans le paysage mais qu'en sera-t-il demain pour ceux de Hayange?

\section{Le territoire d'une « résilience contrariée » par de nombreux handicaps}

16 L'après 2012 et la fermeture des deux derniers hauts-fourneaux lorrains ${ }^{8}$, marquent clairement l'entrée du territoire du Val de Fensch dans un cycle nouveau de transformations, de mutations. La fin de la production ibidem de fonte et d'acier implique, pour les responsables industriels et pour les élus locaux, de favoriser le développement et la modernisation de toutes les unités de l'aval de la société 
relecteur2019-10-04T05:35:00relecteurArcelor-Mittal qui emploie encore actuellement un peu plus de 2000 salariés dans la vallée.

\section{A. Le Val de Fensch est-il un territoire post-industriel ?}

17 C'est précisément ce qui a été inscrit dans l'accord entre le Gouvernement Ayrault et le groupe Arcelor-Mittal, dévoilé le 30 novembre 2012, à savoir investir 180 millions d'euros pour moderniser les unités de laminage à chaud (Serémange-Erzange), à froid (Florange) mais également la cokerie dont la production alimente désormais les hautsfourneaux de Dunkerque. Récemment encore, le groupe Arcelor-Mittal a engagé une réorientation de production de son usine d'électrozingage à Florange laquelle a nécessité 67 millions d'euros supplémentaires. D'ici fin 2019, cette unité ne produira plus que des produits galvanisés destinés au marché automobile à haute valeur ajoutée ${ }^{9}$. La stratégie locale d'Arcelor-Mittal repose dorénavant sur une volonté de renforcer la compétitivité de ses usines en les spécialisant sur des laminages à haute valeur ajoutée pour leur donner de la durabilité.

La situation géo-économique de la vallée de la Fensch n'est en réalité pas totalement comparable avec celle des shrinking cities, villes rétrécissantes ou en déclin (Florentin, 2016), même si certains facteurs sont communs et que ce terme doit faire l'objet d'un regard critique systématique (Fol, Cunningham-Sabot, 2010). Ce terme de shrinking city qualifie en fait « une conjonction de processus relativement connus, mais qui s'observent avec une acuité nouvelle depuis une vingtaine d'années. Il correspond d'une certaine façon à la face sombre de la mondialisation et de la métropolisation, celle des territoires laissés relativement en marge. (...) Une shrinking city est une ville où s'accumulent, avec plus ou moins d'ampleur mais sur un temps relativement long, des processus de déprise démographique, de déclin économique, auxquels s'ajoute souvent une crise des finances publiques locales. Cette conjonction de processus en fait souvent des villes aux services diminués » (figure 2).

Figure 2 - Les spirales négatives des "shrinking cities" (Florentin, 2017)

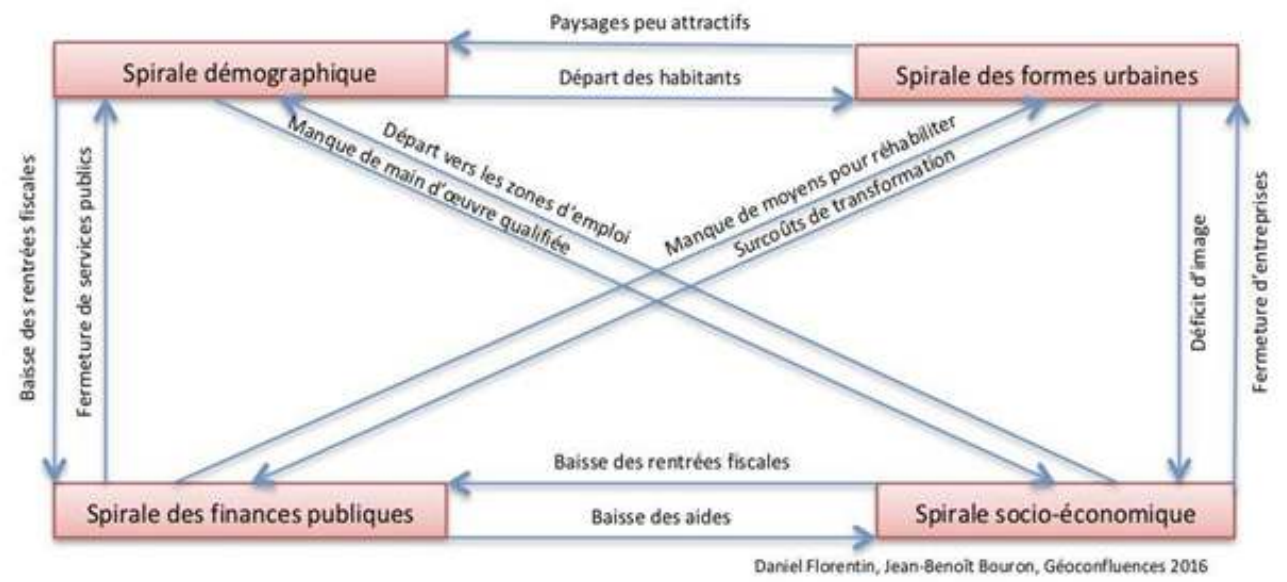

Revue Géographique de l'Est, vol. 58/3-4 | 2018 
Figure 3 - Le modèle des "spirales" appliqué au Val de Fensch est doublement influencé par des héritages complexes à gérer et des formes de "renouveau" impulsé par la dynamique luxembourgeoise

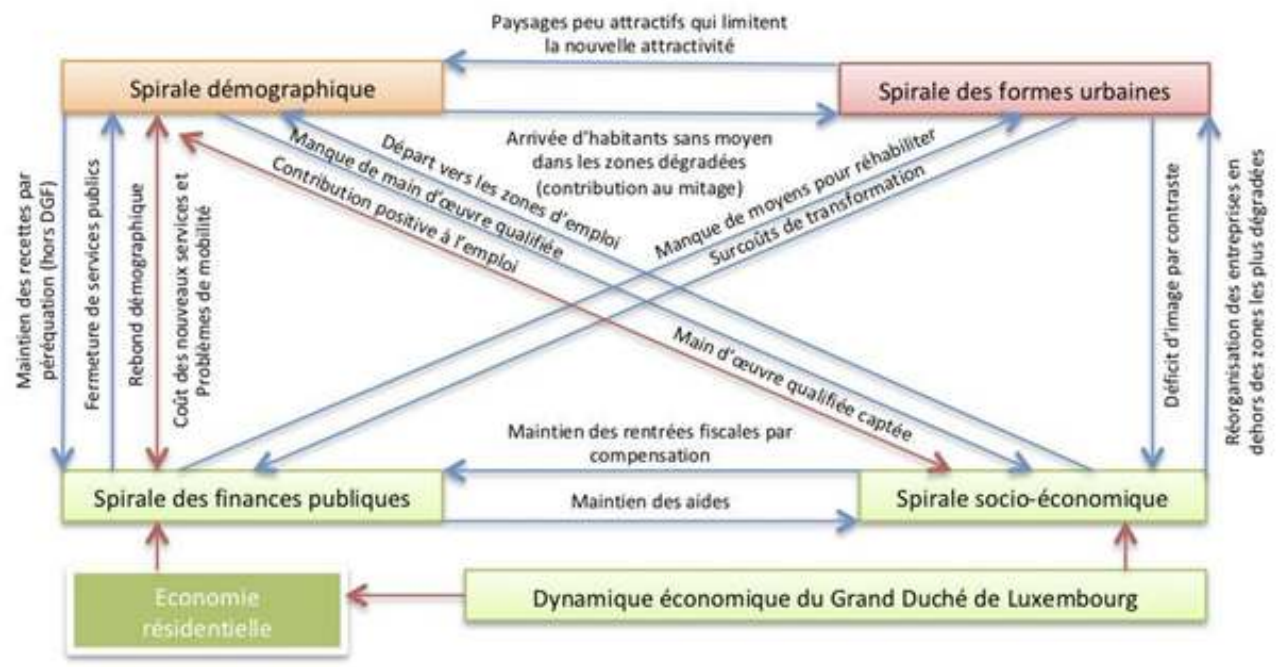

En réalité, cette vallée souffre d'une image ternie tout à la fois par des réalités postindustrielles réelles, marquées et indéniables (Raggi, 2019), mais également par un regard porté sévère et orienté par les tenants du déclinisme, du fatalisme ou par les amateurs d'une littérature misérabiliste annihilant toute volonté de résilience. Deux exemples parmi tant d'autres car ils sont légions, pour bien comprendre ce phénomène auquel est confronté ce territoire. D'abord celui d'un ouvrage intitulé Tour de France des villes incomprises (Noyaux, 2016) : « Knutange, Nilvange, Hayange, Serémange-Erzange, Uckange... Les gens du coin parlent de la vallée des anges - des anges à qui on a collé des plumes et du goudron. Pauvre Fensch! L'homme lui a fait subir les pires outrages : cheminées d'usines, hangars, bâtiments de l'industrie lourde à perte de vue, autoroutes et chemins de fer, cités ouvrières tentaculaires. (...). Sacrifiée sur l'autel du progrès, la petite vallée de la Fensch est morte pour l'industrie, comme les villages détruits de Verdun sont morts pour la France ».

Après une telle description, en partie exacte il est vrai, difficile de parler d'une terre où l'on a envie d'habiter, cette "envie" constituant un moteur déterminant de l'économie résidentielle. Les clichés ont la vie longue. Pire, la vallée de la Fensch est même devenue un moyen littéraire de qualification métaphorique de l'endroit où l'on n'a pas envie de vivre. Ainsi dans un roman policier récent (Permingeat, 2017), l'auteure, faisant parler un de ses personnages, ose : «C'est sinistre, ça me rappelle le bassin minier de la Fensch où je suis allée faire un reportage lors des manifestations des veuves de mines. Ce sont des endroits où on n'aimerait pas vivre».

De même, avec la "goncourisation » de l'auteur lorrain Nicolas Mathieu (2018) dont l'œuvre primée prend pour toile de fond romanesque une vallée désindustrialisée ressemblant beaucoup à notre sujet d'étude, cette question de l'image territoriale fenschoise est encore très récemment revenue sur le devant de la scène médiatique (Bouvarel, 2019 ; Carillet, 2019). Au-delà de la qualité sur le fond et la forme du roman sur lesquels il ne nous appartient pas ici de disserter, les jurys du Goncourt ayant fait leur choix, ce livre a remis en débat, en particulier localement, cette question de l'impact des écrits sur la désindustrialisation en terme d'image et donc d'attractivité des territoires. Pour les uns, cela contribue à ternir plus encore un territoire en transformation permanente depuis 40 ans. Pour eux, cela nuit à son attractivité et à sa 
résilience, notamment quand celle-ci s'appuie sur l'économie résidentielle. Qui voudrait venir habiter dans des lieux dépeints aussi sombrement? Pour les autres au contraire, cette mise en lumière est une aubaine, un moyen de regarder autrement un territoire, de le faire connaître différemment. Ces deux approches peuvent paraître de prime abord antagonistes. Pourtant, en y réfléchissant bien l'une n'exclut pas l'autre à condition de porter un regard objectif et assumé de son propre territoire.

\section{B. Une économie résidentielle en rebond mais encore hésitante}

Comme l'ensemble des territoires frontaliers du Grand-Duché de Luxembourg, le territoire du Val de Fensch connaît un rebond démographique assez marqué depuis une dizaine d'années. En cela, passés les traumatismes successifs, la décroissance urbaine du Val de Fensch n'apparaît plus aussi inéluctable. Le modèle des spirales est en effet ici modifié (figure 3), depuis presque 20 ans, par l'essor du travail frontalier vers le GrandDuché de Luxembourg. Comme l'ensemble des Etablissements Publics de Coopération Intercommunale (EPCI), le long et à proximité des frontières avec ce pays, le Val de Fensch n'a cessé de voir progresser sa population de navetteurs. Les derniers chiffres indiquent ainsi que $34 \%$ des actifs résidant dans les 10 communes du territoire du Val de Fensch travaillent au Grand-Duché de Luxembourg, attirés par un nombre d'emplois à pourvoir plus important et par des conditions salariales et sociales nettement plus intéressantes qu'en France. Ce taux augmente à mesure que l'on se rapproche de la frontière, il dépasse ainsi les 70\% pour la Communauté de Communes du Pays-Haut Val d'Alzette et les 60\% dans la Communauté de Communes de Cattenom et Environs. Dans la Communauté d'Agglomération Portes de France Thionville, limitrophe de celle du Val de Fensch, les travailleurs frontaliers représentent $37,2 \%$ des actifs.

Pour autant, l'économie résidentielle du Val de Fensch ne semble pas bénéficier, tout au moins avec la même intensité, que celle constatée dans les intercommunalités contigües, du développement du travail frontalier. À cela, plusieurs explications qui tiennent pour l'essentiel à un problème récurrent d'image territoriale dégradée en particulier dans la partie amont de la vallée, là où le parc de logements est plus ancien, plus dégradé (AGURAM, 2015) et où les taux de vacances sont largement plus élevés ${ }^{10}$ (figure 4), là où les friches industrielles et minières sont plus proches du tissu urbain existant et pas encore transformées, mais également là où les contraintes urbanistiques et d'adaptation à la nature des sols sont plus lourdes et nécessairement plus coûteuses. S'ajoute à cela dans les villes inscrites en fond de vallée ou sur ses flancs (Algrange, Nilvange, Knutange, Hayange), la raréfaction du foncier mobilisable. Dans ces communes, seules des opérations complexes à porter, de renouvellement urbain pourront permettre de poursuivre le développement de l'économie résidentielle, faute de quoi les fractures territoriales internes au Val de Fensch ne feront que s'accroître. 
Figure 4 - La communauté d'agglomération du Val de Fensch : un territoire aux problématiques multiples

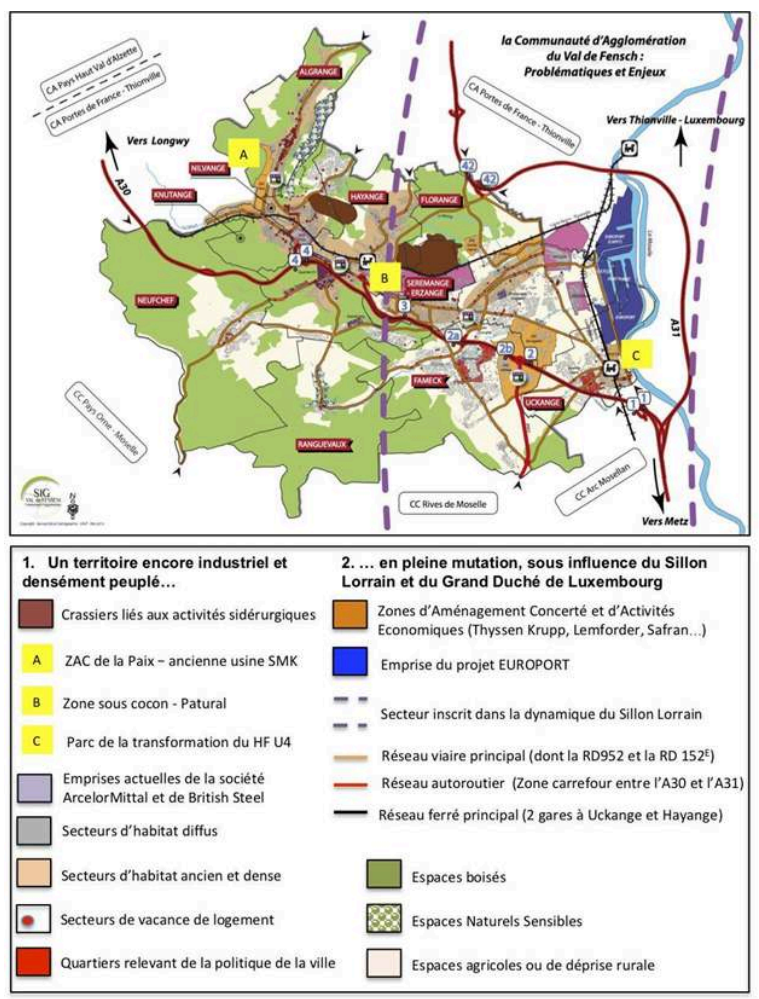

En réalité, comme le relève M. Talandier (2012) en écho aux travaux de L Davezies (2008) sur la théorie des bases et le fonctionnement des économies locales, «l'enjeu résidentiel est l'un des piliers du développement local qui dépend de l'attractivité des territoires, de leurs atouts paysagers, de leurs aménités environnementales ». Or dans le cas des communes du Val de Fensch, si les fonctions productives sont en partie préservées, il n'en n'est de même pour les fonctions de redistribution (services publics rationalisés, démographie médicale compliquée...), de mobilité (secteurs enclavés, manque d'infrastructures notamment en matière ferroviaire...), de consommation (secteur commercial en difficulté en particulier au cœur des villes...). Dans ce que Laurent Davezies appelle en 2014 la circulation invisible des richesses, le système socioéconomique du Val de Fensch apparaît très désavantagé par rapport aux autres territoires frontaliers $\mathrm{du}$ nord Lorrain, tous concernés par une dynamique transfrontalière d'aspiration vis-à-vis du Grand-Duché de Luxembourg (Agences d'Urbanisme du Grand Est, 2018). Ces autres territoires connaissent en effet une situation beaucoup moins dégradée sur le plan de l'image territoriale et s'inscrivent plus dans les logiques de développement et de mobilité qui caractérisent le Sillon Lorrain. Pour reprendre la théorie des bases (Davezies et Talandier, 2014), les territoires du Nord Lorrain sont en quelque sorte spécialisés dans la captation de l'économie résidentielle et tout particulièrement de l'économie issue des travailleurs pendulaires. Le premier janvier 2019, huit intercommunalités situées le long de la frontière Luxembourgeoise, représentant 330000 habitants, ont d'ailleurs créé un Pôle Métropolitain Frontalier ${ }^{* 11}$ pour faire valoir cette spécificité, défendre et mettre en cohérence des politiques publiques pour favoriser cette économie résidentielle, à l'instar de ce qui a été réalisé par le Pôle Métropolitain du Grand Genevois. Dans le cas $\mathrm{du}$ Val de Fensch, on peut ainsi considérer que les fonctions productives sont encore 
importantes mais les héritages industriels limitent la captation des flux de l'économie résidentielle (tourisme et pendulaires notamment). En revanche, les revenus des transferts sociaux (RSA, indemnisation chômage, minimum vieillesse, allocation adulte handicapé...) y sont surreprésentés dans la plupart des communes fenschoises, ce qui indique un déficit d'attractivité territoriale.

La Communauté d'Agglomération, consciente de ses désavantages compétitifs, de ses déterminismes historiques et géographiques, en plus des aménités environnementales qu'elle cherche à développer (création de parcs communautaires, mise en place d'un plan de paysages, d'aides importantes à la réhabilitation du bâti tant sur le plan esthétique que thermique, mobilité douce, protection et mise en valeur du patrimoine, tourisme industriel et marque locale...), a néanmoins choisi, pour favoriser son économie résidentielle, moteur complémentaire des activités industrielles et commerciales, de faire entrer dans son champ de compétences, optionnelles et facultatives, des politiques publiques plus orientées vers les services à la famille. Le développement des espaces multi-accueil pour la petite enfance ou encore l'aménagement numérique du territoire pour donner la possibilité à tous les foyers de pouvoir accéder à internet (et ses services) à très haut débit, entrent dans l'idée que le choix de l'implantation résidentielle se construit aussi autour d'aménités aujourd'hui nouvelles mais demain probablement obligatoires.

Dans cette compétition de l'économie résidentielle qui ne dit pas son nom, il apparaît néanmoins que la vallée de la Fensch possède encore l'atout d'un coût locatif et d'acquisition du foncier bien inférieur à celui des intercommunalités de la première ou de la seconde couronne frontalière (figure 5).

\section{Renouveler, réparer, réorganiser le territoire pour retrouver une attractivité homogène : les défis du Val de Fensch.}

27 La Communauté d'Agglomération du Val de Fensch, dans le cadre de son projet de territoire 2014-2020 (CAVF, 2015), a fait de la transformation de ses espaces de déprise, de ses béances paysagères une priorité de ses actions, considérant que l'image territoriale était un facteur essentiel de son attractivité. Pour cela, elle s'est engagée dans un processus de revitalisation sur le double plan de l'image urbaine et de la reconquête des espaces en friche qui occupent le plus souvent le centre des villes, le tissu urbain s'étant développé autour des usines. Il s'agit donc tout à la fois de mener des opérations pour rétablir les fonctionnalités des espaces occupés par les usines fermées et de travailler sur l'image ternie de l'ensemble du patrimoine connexe : centres-villes surdimensionnés par rapport aux réalités économiques d'aujourd'hui, habitat ancien, souvent dégradé et difficile à entretenir, infrastructures nombreuses, enchevêtrées et complexes à restructurer. Le territoire de la Communauté d'Agglomération du Val de Fensch est jalonné par trois grands sites de transformation, en cours, en passe de l'être ou à penser dans un avenir assez proche. Il s'agit respectivement du projet Evol'U4 à Uckange, du site de la Paix sis sur les bans communaux de Algrange, Nilvange et Knutange et enfin le secteur de Patural à Hayange sous cocon. Nous avons choisi ici d'analyser l'évolution de ces espaces de grande dimension (plus de 100 ha au total) car il s'agit de trois hauts-lieux de la vallée, emblématiques des grandes sociétés sidérurgiques dont certaines ont disparu, la 
Société Métallurgique de Knutange et Lorfonte, et une autre subsiste, Arcelor-Mittal. Ce sont des lieux qui cristallisent les regards et qui, en conséquence, deviennent représentatifs du territoire, au point parfois même d'en devenir des marqueurs identitaires.

Figure 5 - Le Val de Fensch dans le marché transfrontalier de la location autour de la métropole luxembourgeoise (Source : AGAPE, 2019)

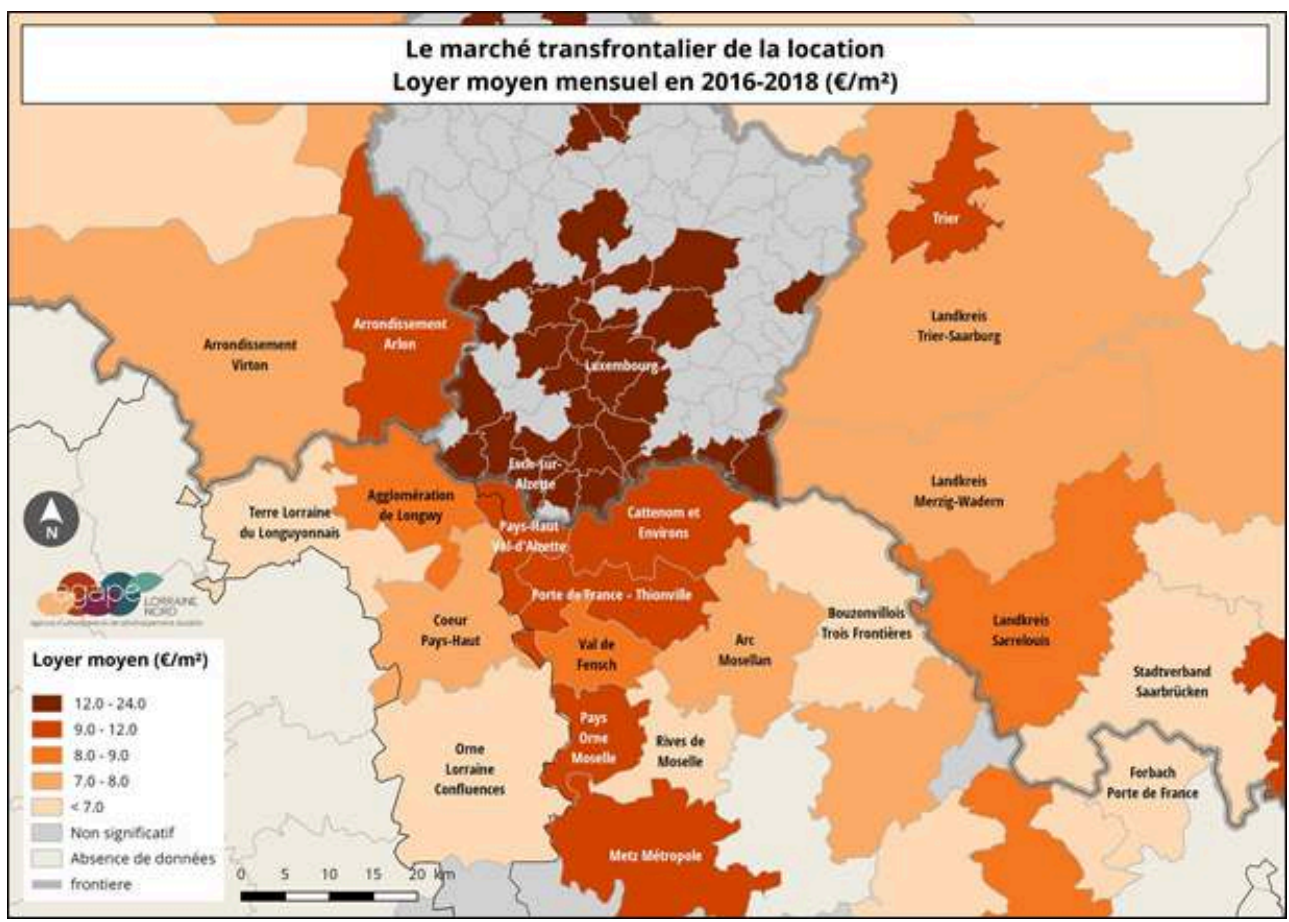

\section{A. Le U4 : un symbole identitaire des territoires industriels en reconversion?}

La reconversion de l'usine à fonte de Uckange doit être regardée à l'aune de ce qui a échoué pour l'usine SMK sur le site de la Paix. En effet, la reconversion de cet espace s'est placée dans une logique de tabula rasa après la fermeture de l'usine en 1983. Tout y a été détruit dans l'objectif d'une réutilisation rapide du foncier qui n'a d'ailleurs pas eu lieu malgré la création dès 1987 du Syndicat Intercommunal d'Aménagement de la Paix (SIASP puis SIAPAX) entre les communes de Algrange, Nilvange, Knutange et Fontoy. A Uckange, au moment même de la fermeture le 17 décembre 1991, l'association MECILOR (MEmoire Culturelle et Industrielle LORraine) milite activement pour le maintien d'une partie de l'usine, misant sur une renaissance immédiate en lieu de convivialité et de culture, dans un refus du fatalisme et du repli sur soi.

Dix années plus tard, en 2001, après de nombreuses études et au terme d'un processus d'acceptation de la patrimonialisation (Edelblutte, 2008), sous l'impulsion de la commune de Uckange et de cette association mémorielle, plusieurs bâtiments de l'ancienne usine, dont notamment l'hyper structure du haut-fourneau U4 sont inscrits à l'inventaire supplémentaire des Monuments Historiques. La Communauté d'Agglomération en devient propriétaire en 2005 et entame dès lors d'imposants travaux de mise en sécurité, de désamiantage, préalable indispensable à l'ouverture au 
public ainsi qu'à la réalisation de la mise en lumière portée par Claude Lévêque, artiste de renommée internationale, intitulée " Tous les Soleils » et inaugurée à l'automne 2007.

30 C'est autour du U4 conservé et artialisé in situ (Roger, 1997 ; Husson, 2018), avec en conséquence des contraintes lourdes vis-à-vis des Architectes de Bâtiments de France (Drouguet et Bodeux, 2017), que vont être agrégés plusieurs projets connexes dans une logique de parc mémoriel à l'image de ce qui a été réalisé en 1999 à Völklinger Hutte ${ }^{12}$ en Sarre, mais également pédagogique, scientifique, économique et culturel. Si la population locale était au départ assez réticente à cette idée d'une patrimonialisation des vestiges sidérurgiques, il semble qu'un retournement se soit opéré, notamment parce que le U4 est devenu une sorte de Landmark identitaire, redonnant fierté aux habitants (Edelblutte, 2016).

31 La plupart des travaux sont menés sous maitrise d'ouvrage de la Communauté d'Agglomération et regroupés dans le projet EVOL'U4 auquel l'Etablissement Public Foncier de Lorraine (EPFL) apporte un soutien financier et d'ingénierie important, dérogeant même à ses règles habituelles de financement qui l'obligent d'ordinaire à être propriétaire du terrain, ce qui n'est pas le cas ici. La ville d'Uckange de son côté crée en 2011, de manière contigüe au parc du haut-fourneau, en lieu et place de l'usine d'agglomération, le Jardin des Traces dont la gestion est assurée par l'association Chrysopée. Ce lieu au pied du U4 rencontre indéniablement un vif succès populaire.

32 Sous l'angle économique, le projet EVOL'U4 peine encore à entrer dans une phase de plein développement tant les coûts de réhabilitation des bâtiments classés autour du U4 (dispatching, sous-station électrique, bâtiment des soufflantes...) sont devenus rédhibitoires dans un contexte de tarissement des finances publiques. En décembre 2013, à l'initiative du Président de la République, François Hollande, a été implantée, dans l'un des bâtiments du projet, une plateforme de recherche portant sur l'économie circulaire des matériaux ferreux au nom de MetaFensch, sous le statut d'un groupement d'intérêt public (GIE) et financé par le Programme d'Investissements d'Avenir (PIA) à hauteur de $20 \mathrm{M€}$. Il s'agit sans conteste d'une véritable locomotive pour l'amorçage économique ${ }^{13} \mathrm{du}$ projet mais également d'une implantation symbolique puisque orientée sur la question de la transformation ${ }^{14}$, thème central de la requalification de la friche des hauts-fourneaux d'Uckange.

De même, plus récemment encore et sur ce même site, Arcelor-Mittal a annoncé son intention de réaliser dans les $2.000 \mathrm{~m}^{2}$ des Grands Bureaux de Uckange, un Digital Lab 4.0 dans l'optique de procéder à une digitalisation de ses processus de production, de faire de la formation de son personnel autour du numérique et d'y faire travailler des start-ups spécialisées dans ces domaines. Pour le leader mondial de la production d'acier, il s'agira tout à la fois de montrer son implication territoriale (en plus de la proximité avec ses usines les plus high-tech et l'accessibilité aisée à la gare de Uckange désormais desservie par 107 TER journaliers) et pour la Communauté d'agglomération un moyen de valoriser ce site dans une perspective plus technologique, disruptive et résiliente. Dans cette planification de restructuration, on peut donc affirmer que le patrimoine industriel peut être utilisé comme un levier d'action stratégique (Del Biondo, 2014). Il permet de soutenir en partie la guérison urbaine, économique et sociale du territoire en fédérant celui-ci autour d'un projet emblématique, attirant potentiellement de nouveaux acteurs socio-économiques (Picon, 2016) et dans des champs nouveaux. 


\section{B. Le site de la Paix : quel renouveau possible près de $\mathbf{4 0}$ ans après la fermeture de l'usine SMK?}

34 La transformation du site de la Paix s'inscrit dans un temps résolument plus long que celui de l'usine d'Uckange. Les premiers aménagements sommaires réalisés in situ, avec l'appui de l'EPFL, datent de la fin des années 80 et furent réalisés par le SIAPAX. Mais la vocation de ce site de 42 hectares, pourtant bien situé à la confluence du ruisseau d'Algrange et de la Fensch, a été fastidieuse à trouver. Il faut dire que les contraintes de sols, tant du point de vue de leur pollution (hydrocarbures, РCB...) que de la fragilité des soubassements, ont obligé à un long travail d'études et d'apurement (Marochini, 2018). La société SMK a certes fait table rase en surface mais n'a quasiment rien extrait de l'architecture souterraine de l'usine, laissant ainsi les fondations, le génie civil des réseaux, les structures de portance, rendant impropre à une reconstruction quasiment l'ensemble pour quelque vocation que ce soit, et en particulier pour l'habitat.

Tous ces vestiges sont autant de contraintes lourdes au réaménagement, y compris sur le plan financier. La Communauté d'Agglomération a récupéré la gestion de la zone en 2002 et y mène depuis des études structurelles et d'anticipation, concomitamment à des travaux de dépollution et d'adaptation des sols. En dehors du secteur haut du site de la Paix, très vite transformé en zone d'activités commerciales et artisanales entre les deux communes de Algrange et Nilvange, sur la partie basse en terme de réalisation concrète à ce jour, seul le parc de la rotonde, dans la partie sud du site à Knutange, a été réalisé et inauguré en 2007. Dans un contexte de tension foncière lié à la pression exercée par le Grand-Duché de Luxembourg et de besoin de développement pour les communes du fond de vallée, la Communauté d'Agglomération, dans le cadre d'une politique volontariste de transformation de ses friches, s'est engagée dans la transformation complète de cet espace selon trois orientations, en adaptant les possibles aux niveaux de pollutions constatées.

Le projet d'aménagement en matière d'habitat, d'environnement et de commerce prévoit désormais plusieurs secteurs distincts, permettant une requalification et une structuration cohérentes de l'ensemble du site. La partie nord de la ZAC de la Paix sera ainsi dédiée à la création d'un nouveau quartier, en couture urbaine avec l'existant d'Algrange, de près de 500 logements. Il proposera une diversité de typologie de logements (petits collectifs et pavillons individuels) mais participera aussi à la promotion de la mixité sociale et s'inscrira dans une démarche environnementale. À cet effet, il comprendra $30 \%$ de logements sociaux et $5 \%$ de logements à prix maîtrisés pour l'accession à la propriété par les personnes âgées. L'idée est de permettre aux habitants de vivre dans un quartier conçu selon les principes du développement durable, favorisant notamment les modes de déplacement doux et la performance énergétique des constructions. Par ailleurs, deux hectares de la partie centrale sont d'ores-et-déjà mis à disposition de l'IUT Thionville-Yutz dans le cadre d'un projet scientifique de dépollution du sol par les plantes selon la technique de la phytoremédiation (miscanthus giganteus...) ${ }^{15}$. Le côté ouest deviendra quant à lui un lieu fédérateur et de sociabilité grâce à une plaine événementielle commune aux villes d'Algrange, Knutange et Nilvange. Enfin, la partie la plus au sud accueillera un secteur d'activités commerciales et artisanales plus moderne. 
37 La procédure ZAC a été menée selon une logique originale de démocratie participative, pour associer les habitants des tissus urbains connexes à la réflexion, en présence du cabinet d'urbanisme lyonnais OBRAS choisi par la CAVF, en tant qu'Assistant à Maîtrise d'Ouvrage (AMO), pour échafauder le projet global. L'aménageur a été retenu par le délégataire selon la méthode du dialogue compétitif dont l'objectif est actuellement de produire un avant-projet détaillé d'aménagement aux élus communautaire. Cet espace pourrait ainsi connaître ses premiers développements urbains concrets d'ici 2 à 3 ans. Cette évolution retracée rapidement montre bien toutes les difficultés inhérentes à la transformation des friches industrielles, en particulier là où les usines étaient le cœur des villes.

\section{Patural : un espace à fortes potentialités dans le cœur historique de Hayange et de la vallée de la Fensch}

38 Il aura donc fallu 40 ans pour que le Site de la Paix trouve le chemin de nouvelles fonctionnalités, après moins d'un siècle d'exploitation industrielle. Gageons qu'il n'en sera pas de même pour le site Patural à Hayange, et qu'ici prévaudra une logique d'anticipation. Ce troisième grand site industriel du territoire du Val de Fensch correspond aux actuels hauts-fourneaux, à l'aciérie et à la coulée continue (Figure 6) dont l'activité a été arrêtée fin 2012 et dont ArcelorMittal a finalement annoncé le démantèlement fin 2018 à « l'issue des conflits sociaux les plus spectaculaires du début du XXI ème siècle» (Cosnard, 2018). Il faut dire que peu nombreux étaient ceux qui croyaient en l'hypothèse d'un redémarrage. Une telle ambition aurait impliqué un investissement financier colossal alors que la méthode logistique d'alimentation des laminoirs à chaud de Serémange depuis Dunkerque semble montrer une double efficacité sur les plans technique et financier puisque la Contribution à la Valeur Ajoutée de l'Entreprise ${ }^{16}$ réglée annuellement par Arcelor-Mittal à la Communauté d'Agglomération du Val de Fensch est restée stable voire à progresser, dans un contexte économique générale assez fluctuant ces dernières années. L'emprise industrielle de cet espace est de 52 hectares. Son positionnement géographique en fond de vallée, en entrée de ville de Hayange, établissant une jonction possible entre les versants sud et nord, constitue un lieu des possibles à fort potentiel alors que la plupart des communes sont en recherche de foncier pour le développement de leur économie résidentielle.

De surcroît, la position inclusive au tissu urbain actuel pourrait permettre de définir des nouvelles connexions viaires permettant une meilleure desserte d'un secteur de cette ville aujourd'hui encombré et sans véritable lisibilité urbaine. Si l'on y ajoute les terrains contigus de la platinerie situés eux-aussi en entrée de ville qui pourraient doublement servir à des fonctions économiques et de rétention hydraulique que les récents débordements de la Fensch rendent d'autant plus nécessaire, la proximité de la gare de Hayange qui doit impérativement retrouver un second souffle, la réalisation future d'une ligne de Bus à Haut Niveau de Service (BHNS) dont le pôle d'échange avec tout le haut de la vallée doit être réalisé sur le secteur Platinerie, la proximité de nombreux équipements publics, en particulier le Centre Hospitalier Metz-ThionvilleBriey-Hayange et le siège de la Communauté d'Agglomération du Val de Fensch, mais également des respirations vertes à valoriser notamment au travers d'un parcours piéton le long de la Fensch reliant le centre-ville de Hayange et le Parc de l'Orangerie, on mesure toutes les potentialités de développement de ce site. 
Bien entendu, les écueils de la mutation attendue sont nombreux, en particulier en matière de négociation foncière et de dépollution avec Arcelor-Mittal qui a déjà indiqué que la remise en état du site serait réalisée, comme la législation le prévoit, pour un usage industriel. Le reste à charge pour la collectivité pour l'acquisition et l'adaptation des terrains à de nouveaux usages risque d'être considérable. En plus, le réaménagement et la restructuration des réseaux, notamment ferrés, ainsi que le portage administratif, technique et financier de la transformation de ce site seront indéniablement très complexes à mener.

Figure 6 - Le site de Patural à Hayange, un secteur industriel sous cocon aux potentialités urbaines exceptionnelles

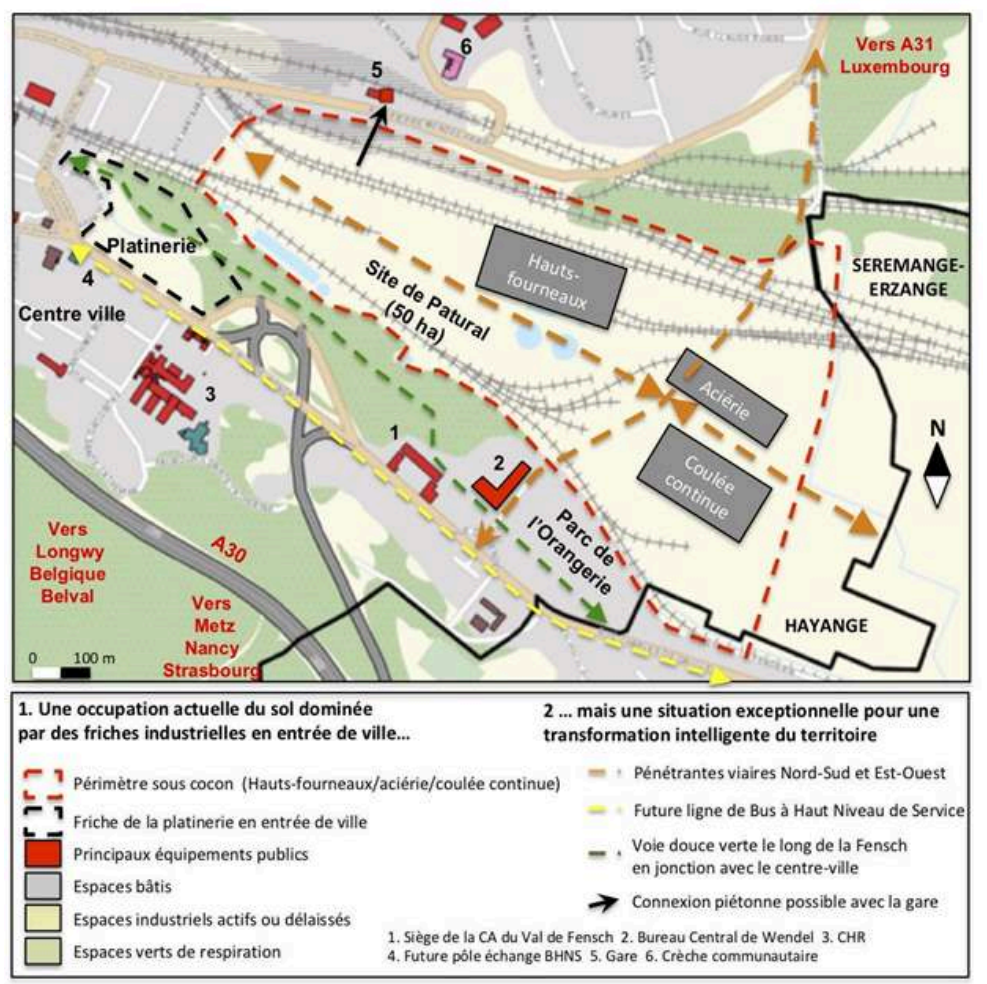

41 Mais réfléchir et anticiper le devenir de ce site demeure un impératif. Ceci ne pourra être mené que par la Communauté d'Agglomération du Val de Fensch qui réfléchit à la mise en œuvre d'une Grande Opération Urbaine (GOU) ${ }^{17}$ et un Plan Partenarial d'Aménagement (PPA), tels que prévus par la loi n²018-1021 du 23 novembre 2018 portant Evolution du Logement et Aménagement Numérique (ELAN). En effet, au regard des enjeux financiers et d'ingénierie territoriale liés à cette ambition, aucune commune prise isolément ne pourrait y parvenir.

La complexité du sujet est telle que le président de l'intercommunalité a même d'oreset-déjà interpellé l'Etat pour que des moyens exceptionnels tant financiers qu'en matière d'ingénierie soient dégagés. Alors qu'Arcelor-Mittal a déjà débuté le processus de déconstruction de ses installations classées, la communauté d'agglomération sollicite en effet la conduite d'une expertise de niveau international convoquant une ingénierie hautement spécialisée dans le cadre du programme "Territoires d'Industries " lancé par le Gouvernement à l'occasion du Conseil de l'Industrie le 22 novembre 2018. Le coût d'une telle étude étant considérable, l'intercommunalité va également étudier la possibilité de mobiliser des financements de fonds spécialisés dans 
la transformation des friches industrielles tels que Ginkgo et Brownsfields ${ }^{18}$, en lien avec Arcelor-Mittal ou par appel à projet de l'Agence De l'Environnement et de la Maîtrise de l'Energie (ADEME).

De même, la réussite d'un tel projet nécessite également, et peut être essentiellement, une acceptabilité sociale et une forme "d'unanimisme" politique sur le long terme. L'implication de la population locale dans ces réflexions autour de la transformation des usages de l'espace est également primordiale, faute de quoi il sera difficile d'en faire admettre les enjeux, les ressorts et les implications. Les réflexions engagées sur ces sujets par l'Université Populaire du Val de Fensch ${ }^{19}$, en lien avec la Communauté d'Agglomération, s'inscrivent pleinement dans cette idée d'associer la population quant aux usages futures possibles de son territoire. La question de la patrimonialisation ou non des hauts-fourneaux de Patural va se poser mais force est de constater qu'aujourd'hui celle-ci ne semble pas prioritaire. Peu de voix se manifestent en effet en ce sens.

Sur le plan politique, l'aménagement d'un tel espace, aussi parce que totalement inscrit sur le ban communal de la ville de Hayange dont la majorité Front National en place depuis le dernier renouvellement municipal de 2014 ne fait pas montre d'un intérêt manifeste en matière de transformation de la vallée en général et de la ville en particulier, nécessitera de trouver un accord entre toutes les villes de la communauté d'agglomération. Il s'agira dès lors de le placer dans une logique de long terme, de continuité, une forme de pacta sunt servanda ${ }^{20}$ qui lie les parties dans un projet de territoire plus que dans une ambition politique.

\section{Des politiques locales interventionnistes pour repenser les villes industrielles de la vallée de la Fensch et permettre leur résilience urbaine}

45 La vallée de la Fensch tente de se réinventer, nous venons de le voir, par la transformation de ses friches et en particulier des plus importantes d'entre-elles, mais pas seulement. A ces requalifications zonales très perceptibles néanmoins dans le milieu urbain, l'intercommunalité a ajouté récemment des dispositifs nouveaux visant à changer là encore le regard porté sur le territoire. Ils s'inscrivent dans une logique spatiale différente s'attachant à une transformation tantôt linéaire tantôt pointilliste, avec des échelles d'actions différentes.

L'opération Coeur de Villes Coeur de Fensch (CVCF) entre dans la première catégorie (figure 7). Son objectif est de requalifier les routes départementales 952 et $152 \mathrm{e}$ qui traversent le territoire depuis Uckange jusqu'à Algrange lesquelles constituent une véritable "Avenue de la Fensch ", pour donner à celle-ci une identité, une unité, une cohérence de conception (mobilier et matériaux urbains...), bref un cachet et une image plus attractive.

47 Ce projet ne se limite toutefois pas qu'aux aspects routiers. Il s'agit en effet de conduire plusieurs actions dans des champs de compétence transversaux (mobilité douce, projet de BHNS porté par le SMITU ${ }^{21}$ à l'échelle du Périmètre de Déplacement Urbain (PDU), campagne de ravalements esthétique et thermique des façades, mise en valeur paysagère, enfouissement des réseaux...) autour de cet axe fédérateur où se concentrent les fonctions commerciales et tertiaires mais également de créer, au long cours, des espaces de respiration urbaine (parcs paysagers ${ }^{22}$, placettes aménagées, 
travail qualitatif sur les entrées de villes...) (CAVF, 2015). Ce programme d'actions, impulsé sous cette forme dans le mandat en cours, s'inscrit dans un temps long (a minima une dizaine d'années) compte tenu de l'ampleur des travaux à mener (44 tronçons - 24 kilomètres de voirie devenue communautaire) et des coûts financiers, lesquels devraient dépasser au final les 40 millions d'euros.

Dans les centres des villes de la vallée, et en particulier dans la partie amont dont nous avons à plusieurs reprises souligné les singularités, des actions plus pointillistes et sériées sont en passe d'être menées et devraient entrer en phase opérationnelle d'ici peu. Il s'agit ici d'endiguer la dévitalisation plus marquée de ces communes aux quartiers très anciens ainsi que la dépréciation progressive qui concerne les commerces, les services, l'attractivité de l'habitat et la vie du bourg dans sa globalité (AGURAM, 2017). Cette tendance observée à Algrange, Nilvange et Knutange a amené les élus communautaires à engager très récemment une étude à vocation transversale, en lien avec l'Etablissement Public Foncier de Lorraine (EPFL), pour organiser la résistance face à cette évolution constatée et trouver les ressorts de son inflexion.

Figure 7 - Un exemple de transformation ante post de l'image urbaine : l'opération Cœur de Villes, Coeur de Fensch, à Algrange (travaux en cours d'achèvement)

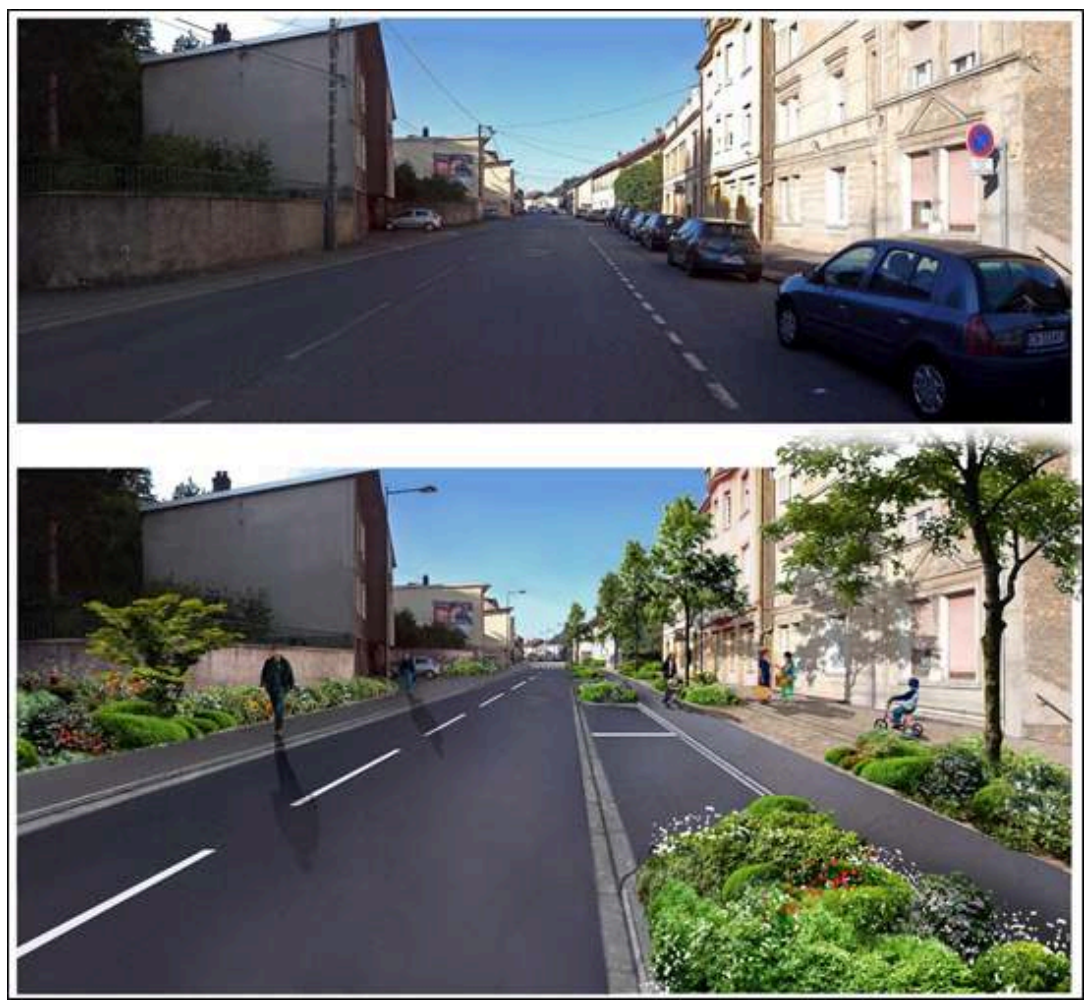

Source: Communauté d'Agglomération du Val de Fensch (2016) - Rue du Général de Gaulle (Partie N)

49 Cette étude encore en cours, réalisée par le cabinet d'architectes Urbicus pour le compte de la Communauté d'Agglomération du Val de Fensch, aurait pu s'inscrire dans le cadre des financements du programme Coeur de Villes initié le 27 mars 2018 par le Ministre de la Cohésion des Territoires, Jacques Mézard, mobilisant 5 milliards d'euros sur 5 ans pour redonner de l'attractivité et de la vitalité aux centres des villes, notamment moyennes, mais il n'en n'a rien été. Dans le bassin de vie, seule la ville de Thionville, ville-centre de la Communauté d'agglomération Portes de France Thionville, 
a pu bénéficier de ce dispositif alors que la situation des centres villes de la Communauté d'agglomération du Val de Fensch, notamment à Hayange, est bien plus dégradée.

L'objectif est ici de développer une approche globale, à l'échelle de trois communes, mobilisant tous les acteurs concernés sur des périmètres précis, à l'échelle de l'îlot urbain, avec des outils opérationnels d'action et de financement. S'agissant des problématiques de l'habitat (plus de 50\% des logements dans ces trois communes ont été construits avant 1949), il s'agira, après la phase diagnostic qui s'achève (figure 8), d'améliorer les conditions générales et de lutter contre une vacance très élevée autant qu'un habitat fortement dégradé (la multiplication des copropriétés dégradées inquiète les acteurs locaux) ou de recycler, tant que faire se peut, un foncier mobilisable très dispersé. Une requalification diffuse des espaces publics, en complément à celle déjà engagée avec le programme Cœur de Villes Cœur de Fensch, devra être menée au risque d'un traitement trop différencié des espaces urbains.

Figure 8 - Le parc intercommunal des deux vallées, support de la redynamisation des centres bourgs de Algrange, Nilvange et Knutange

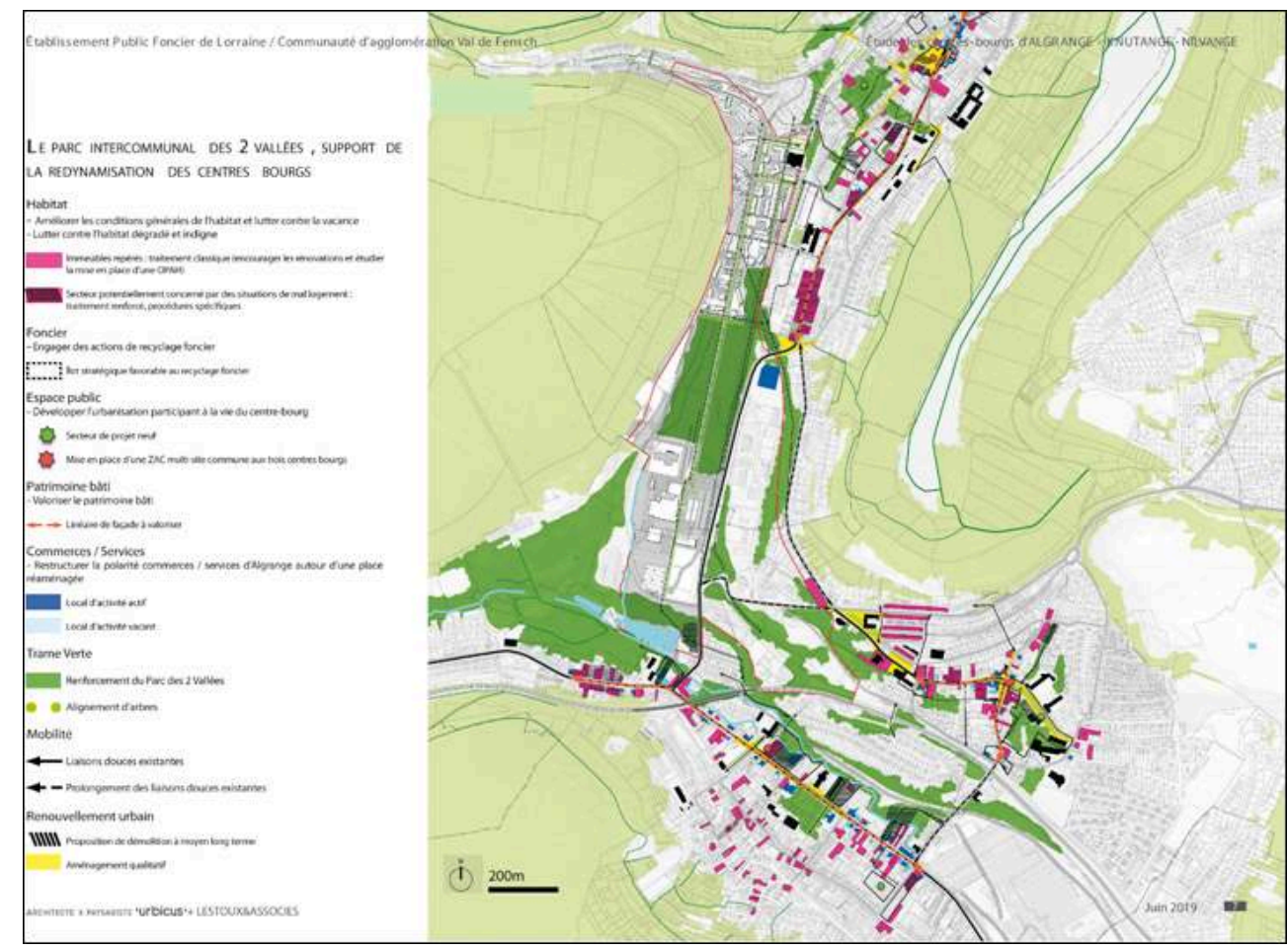

Source: Cabinet Urbicus, 2019

51 Il en est de même sur le plan commercial tout à la fois en difficulté et littéralement émietté dans ces trois communes (figure 8). À ce sujet, il est fondamental de chercher à favoriser des nouvelles polarités commerces-services et éviter une concurrence infraterritoriale sans avenir. À l'instar de ce qui a pu être mené dans d'autres villes en France, la mise en œuvre d'un "remembrement commercial", instrument foncier ancien (Tristan, 2017) mais en plein renouveau, pourrait permet d'accroître les surfaces grâce à des opérations de préemption et des projets de réhabilitation. L'idée est de regrouper de petits espaces commerciaux pour en créer de plus grands afin de moderniser l'armature commerciale, en tenant compte des situations locales. Il s'agit 
certes d'un travail de long terme, nécessitant de multiples partenariats, avec les propriétaires en premier lieu, mais également une forme d'union sacrée politique locale, pouvant donner lieu à des résultats intéressants.

Cette méthode de travail dans ces trois villes est d'autant plus nécessaire qu'avec le développement du site de la Paix établissant un lien entre celles-ci (figure 8), se dessine une difficulté supplémentaire, à savoir un transfert possible des habitants vers l'habitat récent au détriment de l'ancien. Le développement urbain pourrait ainsi concourir, « en boomerang ", à accélérer le processus de dévitalisation des centres historiques.

\section{Conclusion}

Le territoire du Val de Fensch, juxtaposition de villes-usines, a connu et connaît encore de profondes métamorphoses. Avec les récents conflits sociaux très médiatisés autour de la mise sous cocon et finalement fermeture définitive de la dernière filière continentale française de production d'acier et des scrutins locaux successifs placés sous le signe du déclin (Berdenet, 2014), une nouvelle ère s'ouvre encore, avec des permanences industrielles fortes : ArcelorMittal (2000 salariés), Thyssen Krupp (1300 salariés), British Steel Rail (500 emplois), Lemforder (300 salariés)..., qui en font une vallée très différente de la très proche vallée de l'Orne où la page de l'usine a été totalement fermée (Mathis D. et Mathis A., op. cit.), mais également le besoin d'une transformation des espaces en friche et d'un renouvellement urbain d'ampleur.

Il s'agit d'impératifs pour que le territoire puisse retrouver de l'attractivité, une économie résidentielle stable et s'inscrire ainsi pleinement dans la dynamique territoriale de la partie nord du Sillon Lorrain, avec comme métropole de référence le Grand-Duché de Luxembourg. Cette tâche qui incombe pour l'essentiel au principal acteur de l'aménagement du territoire, en l'espèce l'intercommunalité, n'est pourtant pas sans écueils tant la conjugaison des enjeux humains, environnementaux, sociaux, économiques, patrimoniaux et financiers est ici particulièrement complexe.

Dans ce contexte, nous avons pu montrer qu'une forme de résilience territoriale existait néanmoins pour impulser les transformations nécessaires et que les acteurs politiques et de la société civile s'inscrivaient en grande partie dans cette logique. Le jeu d'acteurs territoriaux, autrefois centré sur des logiques strictement communales où chacun voulait faire table rase du passé, s'est progressivement ouvert sur l'idée que les friches industrielles n'étaient pas que les symptômes visibles de la fatalité. Elles ne sont pas non plus nécessairement des espaces en marges, sujet géographique d'actualité, et peuvent aussi être considérées comme des marges de manœuvre pour l'aménagement d'un territoire (Janin et Andres, 2008), des laboratoires pour la transformation des villes (Vaneeckout, 2017), des espaces permettant le recyclage d'un foncier pour épargner les terres agricoles trop facilement urbanisées.

Les singularités du Val de Fensch, son histoire sidérurgique inachevée, son futur encore industriel, ses mutations permanentes, en font un champ d'études plus qu'original. Elles méritent néanmoins de venir en comparaison externe avec d'autres territoires et en évaluation interne notamment sous l'angle de leur coût économique dans un contexte d'assèchement des finances publiques. Une comparaison qui doit être géographique, à l'échelle de la Grande Région (Sarre, Rhénanie- Palatinat, Belgique, Grand-Duché de Luxembourg, Région Grand Est française), avec d'autres systèmes 
politiques, d'autres pratiques et cultures de l'aménagement du territoire, d'autres regards sociaux sur la mutation des paysages industriels.

De même, les politiques publiques menées par le Val de Fensch dans ce champ d'intervention doivent être confrontées à l'avis des populations locales tant du point de vue du réalisé que de ce qu'il reste encore à faire. Pour anticiper les mutations de demain, il faut changer de paradigme, pour que les populations regardent autrement leur territoire et qu'une large adhésion sociale, politique et économique se dessine autour de cette ambition.

\section{BIBLIOGRAPHIE}

AGence d'URbanisme des Agglomérations de Moselle (AGURAM), 2015, Programme Local de l'Habitat de la Communauté d'Agglomération du Val de Fensch: bilan à mi-parcours, $92 \mathrm{p}$

Agences d'urbanisme du Grand Est, 2018, Enjeux et défis du transfrontalier (SRADDET Grand Est), 90 p. AGence d'URbanisme des Agglomérations de Moselle (AGURAM), 2017, Ars-sur-Moselle : étude de dynamisation du centre bourg: synthèse de la démarche, $31 \mathrm{p}$.

Berdenet C., 2014, «À Florange après la crise ArcelorMittal, un scrutin sous le signe du déclin », Le Monde, 17/03/2014.

Bouvarel L., « Nicolas Mathieu : un Goncourt sous le haut-fourneau », Le Républicain Lorrain, 25 juin 2019.

Cabinet Urbicus, 2019, Étude des centres bourgs d'Algrange, Knutange, Nilvange : référentiel foncier et fiches actions, $35 \mathrm{p}$.

Carillet JB., « En Moselle, le haut-fourneau classé de Uckange accueille le Goncourt », le Figaro, 27 juin 2019.

Communauté d'Agglomération du Val de Fensch (CAVF), 2015, Projet de territoire 2014-2020, 96 p.

Cosnard D., 2018, "ArcelorMittal ne veut pas rallumer les hauts-fourneaux de Florange », Le Monde, 13 décembre 2018.

Crouzet F., 1997, « Naissance du paysage industriel », Histoire, économie et société, Environnement et développement économique, p. 419-438, https://www.persee.fr/doc/ hes_0752-5702_1997_num_16_3_1955.

Davezies L., 2008, La République et ses territoires. La circulation invisible des richesses, Paris, Seuil, 110 p.

Davezies L., 2014, La crise qui vient. La nouvelle fracture territoriale, Paris,, Seuil, 110 p.

Davezies L., Talandier M., 2014, L'émergence des systèmes productivo-résidentiels: territoires productifs - territoires résidentiels : quelles interactions ? Rapport du CGET, $136 \mathrm{p}$

Del Biondo L. 2014, Les stratégies de recomposition urbaine soutenable des anciens territoires industrialourbains. Etude du territoire franco-luxembourgeois de la haute vallée de l'Alzette et apports d'exemples européens, Thèse de doctorat en géographie, Nancy, $425 \mathrm{p}$. 
Drouguet N., Bodeux P., 2017, « Vive les hauts-fourneaux ! », Liège, Dérivations hors-série n³, 288 p.

Edelblutte S., 2008, « Paysages et territoires du patrimoine industriel au Royaume-Uni », Revue Géographique de l'Est, vol 48/1-2/ 2008, 29 p.

Edelblutte S., 2016, « Les territoires anciennement industrialisés : du cœur à la marge, de la marge au redéveloppement ? ». In : Woessner R., (dir.), La France des marges, Ed. Atlande, p. 138-151.

Florentin D., 2016, « Shrinking city », Notion à la une de Géoconfluences. http:// geoconfluences.ens-lyon.fr/glossaire/shrinking-city.

Fol S., Cunningham-Sabot E., 2010, « Déclin urbain » et Shrinking Cities : une évaluation critique des approches de la décroissance urbaine », Annales de géographie ${ }^{\circ}$ 674, p. 359-383.

Gehring J-M. Saint-Dizier C., 1983, « Les pays du fer ». In : Frécaut R. - dir., Géographie de la Lorraine, Presses Universitaires de Nancy, p. 433-447.

Gehring JM., 1973, « L'agglomération mono-industrielle de la Fensch : éléments de géographie sociale », Actes du colloque de l'association interuniversitaire de l'Est.

Husson J.-P., 2018, Artialisation, mise en scène des usines et des forges en Lorraine, Conférence présentée à Hayange le 13 mars 2018 à l'Hôtel de communauté de la Communauté d'Agglomération du Val de Fensch.

Husson J-P., 2016, La Lorraine des Lumières : voyage dans les temporalités, les paysages et les territoires, Strasbourg, Editions Vents d'est, 308 p.

Janin C., Andrès L., 2008, « Les friches: espaces en marge ou marges de manœuvre pour l'aménagement des territoires ?", Annales de Géographie, p 62-81.

Jeanneney J.-N., 2019, François de Wendel en République. L'argent et le pouvoir, 1914-1940, Paris, Biblis CNRS, $672 \mathrm{p}$.

Kaercher-Régnery M. et M.-L., 1990, Fensch, vallée du fer: chronique historique, industrielle et minière, Editions Fensch Vallée, 503 p.

Lévy J., Fauchille J.-N., et Povoas A., 2018, Théorie de la justice spatiale : géographies du juste et de l'injuste, Paris, Odile Jacob, $344 \mathrm{p}$.

Marochini É. 2018, « Regard géo-historique sur la difficile transformation des friches industrielles de la vallée de la Fensch : l'exemple de la reconversion en cours du site de l'usine sidérurgique intégrée SMK (1897-2018) », Géocarrefour [En ligne], 92/2 | 2018, mis en ligne le 28 décembre 2018, consulté le 15 juillet 2019. URL : http://journals.openedition.org/geocarrefour/11777 ; DOI : 10.4000/geocarrefour.11777

Marseille J., 2005, Les Wendel : 1704-2004, Paris, Perrin, 348 p.

Mathieu N., 2018, Leurs enfants après eux, Actes Sud, 426 p.

Mathis D., Mathis A., 2017, «Régénération de la conurbation sidérurgique du Val de Fensch », Territoire en mouvement, Revue de géographie et aménagement, 33/2017, https://journals.openedition.org/ tem/4039

Noyaux V, 2016, Tour de France des villes incomprises, Paris, Éditions du trésor, 224 p.

Permingeat E., 2017, Le crime est dans le pré, Éditions nouvelles plumes, 576 p. 
Picon M., 2016, « Le patrimoine industriel de l'U4 au service de la Fensch Valley », The conversation France.

Printz A., 1966, La vallée usinière, Metz, Éditions Le Lorrain, 174 p.

Printz M., 2017, Vallée de la Fensch, vallée du Fer, Collection Les patrimoines du Républicain Lorrain, $51 \mathrm{p}$.

Printz M.,Schmidt L., 2012, Les archives du ciel : usine de la Fensch au temps de l'apogée sidérurgique, Vaux, Editions Serge Domini, 120 p.

Raggi P., 2019, La désindustrialisation de la Lorraine du Fer, Paris, Classiques Garnier, 506 p.

Roger A., 1997, Court traité du paysage, Folio Essais, 249 p.

Sihol B., 2007 « La théorie du patronage de Le Play : une illustration », Revue Française d'Histoire des Idées Politiques 2007/1 ( $\left.{ }^{\circ} 25\right)$, p. 109-124, https://www.cairn.info/revue-francaise-d-histoiredes-idees-politiques1-2007-1-page-109.htm

Talandier M.,2012, L'économie résidentielle à l'horizon 2040. Futuribles, Association Futuribles, p. 35-49.

Tristan J., 2017, L'État, le petit commerce et la grande distribution, 1945-1996: une histoire politique et économique du remembrement commercial, Thèse de Doctorat, Université Paris I PanthéonSorbonne, $495 \mathrm{p}$.

Vaneeckout L., 2017, « Les friches se transforment en laboratoire de la ville », Le Monde, 12/06/2017.

Veschambre V., 2008, Traces et mémoires urbaines, enjeux sociaux de la patrimonialisation et de la destruction, Rennes, PUR, 315 p.

Villar C, et David -M.,. 2014, La résilience : un outil pour les territoires, CEREMA, 8 p, https:// www.cerema.fr/fr/actualites/resilience-outil-territoires.

\section{NOTES}

1. Algrange, Fameck, Florange, Knutange, Hayange, Neufchef, Nilvange, Ranguevaux, SerémangeErzange, Uckange.

2. L'implication et le pouvoir politique de François de Wendel au début du XX ${ }^{\text {ème }}$ siècle furent considérables. Jacques Marseille (op. cit.) parle à son propos et des barons du fer en général : « des maîtres de la France, d'hommes tout-puissants constituant un Etat dans l'Etat, des tireurs de ficelles qui ont transformé en pantins ou homme de paille les hommes politiques les plus importants ».

3. La commune de Fontoy appartient à la Communauté d'Agglomération de Portes de France Thionville alors que la Fensch y prend sa source et que ses habitants s'appellent les fenschois.

4. Partie d'une forge où l'on fend le fer, en barres ou en toute autre forme.

5. Création de complexes intégrés modernes à Dunkerque en 1956 et à Fos-sur-Mer en 1973.

6. Mine la Paix à Algrange : la dernière tournée, Républicain Lorrain, août 1983.

7. En décembre 1991, un concert symbolique sera donné sur le site par le chanteur Bernard Lavilliers. Il y interpréta notamment son morceau « Fensch Vallée » écrit en 1976.

8. Deux hauts-fourneaux sont encore actifs à Pont-à-Mousson mais produisent uniquement de la fonte ductile. Le site, propriété de Saint-Gobain, connait également des difficultés actuellement. Les hauts-fourneaux ont été éteints ponctuellement et la baisse d'activité a amené, fin 2016, le 
groupe Saint-Gobain à solliciter auprès des pouvoirs publics des mesures de chômage partiel pour 750 salariés.

9. Aciers dits USIBOR et DUCTIBOR en grande largeur.

10. Dans ces trois communes (Algrange, Nilvange et Knutange), le taux de vacance des logements dépasse largement la moyenne communautaire (8,7\%). Il est de 13,4\% à Algrange et Knutange, de 10\% à Nilvange. Source : INSEE RP 2009-2014. Rapport AGURAM (2017).

11. Syndicat mixte regroupant des intercommunalités prévu par la loi no 2010-1563 du 16 décembre 2010 de réforme des collectivités territoriales (dite loi RCT). Des compléments ont été apportés par la loi no 2014-58 du 27 janvier 2014 de modernisation de l'action publique territoriale et d'affirmation des métropoles (dite loi MAPTAM).

12. En 1994, l'Unesco reconnaît l'usine sidérurgique et les hauts-fourneaux de Völklingen comme "Patrimoine Culturel Mondial".

13. Le projet EUROPORT (renommé ELOGIN4) et sa future plateforme multimodale (Fer-RouteVoie fluviale), situé à quelques mètres seulement du site $\mathrm{du} \mathrm{U} 4$, peut également constituer un point d'accroche pour un développement économique autour des questions logistiques et de recyclage (projets du syndicat mixte de transport et traitement Des Déchets De Lorraine Nord (SYDELON)).

14. Un projet de «Jardin de la transformation » s'insère dans le projet Evol'U4.

15. La phytoremédiation est un ensemble de technologies utilisant les plantes pour réduire, dégrader ou immobiliser des composés organiques polluants du sol, de l'eau ou de l'air provenant d'activités humaines.

16. Part variable de la Contribution Economique Territoriale (système fiscal qui a remplacé la Taxe Professionnelle en 2010) et liée au chiffre d'affaires localisé de la société.

17. La création et la réalisation des opérations d'aménagement est réputée d'intérêt communautaire. L'établissement public de coopération intercommunale ou la collectivité territoriale portant la GOU est alors compétent pendant toute la durée de la grande opération d'urbanisme pour la réalisation, la construction, l'adaptation ou la gestion d'équipements publics relevant de la compétence de la commune d'implantation, nécessaires à la grande opération d'urbanisme, et identifiés et localisés dans l'acte de qualification. Cet établissement ou cette collectivité assure alors la maîtrise d'ouvrage de ces équipements.

18. Fonds d'investissements privés (Private equity) avec des partenaires publics importants (Caisse des dépôts, Banque Européenne d'Investissement...) qui se sont spécialisés dans la dépollution-transformation des friches industrielles. Des projets pilotés selon cette méthode sont actuellement en cours à Sevran, à Lyon, à Marseille, à Montpellier, à Villeneuve-la Garenne..., après avoir été largement utilisés aux Etats-Unis ou en Grande-Bretagne.

19. L’Université Populaire du Val de Fensch est un mouvement qui cherche à favoriser un débat citoyen dans la vallée de la Fensch, en y associant des chercheurs, des acteurs de la vie associative et politique, des experts autant que des profanes. Elle a initié le 19 novembre 2017, un cycle de débats sur le thème : Réinventer la ville, réenchanter la vie. Quel avenir pour notre héritage sidérurgique?.

20. L'expression pacta sunt servanda est une locution latine signifiant que les conventions doivent être respectées. Pacta sunt servanda implique donc que les parties à une convention doivent respecter les obligations qui en découlent et ne sauraient en aucune manière s'en affranchir.

21. Syndicat MIxte des Transports Urbains, Autorité Organisatrice du Transport du bassin thionvillois.

22. 4 parcs paysagers communautaires ont d'ores-et-déjà étaient réalisés : les parcs de la Rotonde à Knutange, de Sainte-Neige à Hayange/Neufchef, de Sainte-Barbe à Algrange et pédagogique de Ranguevaux. 


\section{RÉSUMÉS}

Dans cet article, l'auteur s'interroge sur les capacités d'un territoire industriel en pleine restructuration, situé dans le nord de l'ancienne Lorraine à proximité de la frontière luxembourgeoise, à se réinventer, à trouver les voies de sa résilience. Après avoir dépeint les héritages, tantôt passifs, tantôt legs, il s'attache à regarder quels sont les facteurs territoriaux, endogènes et exogènes, qui peuvent expliquer ses évolutions en cours, notamment sous l'angle de l'économie résidentielle versus l'économie productive. Il montre enfin, à l'appui de plusieurs projets de transformation territoriale (friches industrielles et urbaines notamment) menés ou en passe de l'être au sein de la Communauté d'Agglomération du Val de Fensch, l'importance d'engager les territoires encore industriels dans une démarche prospectiviste et anticipatrice.

In this article, the author wonders about the capabilities of an industrial area undergoing restructuring, located in the north of the old "Lorraine " near the Luxembourg border, to reinvent itself, to find the ways of its resilience. After describing inheritances, sometimes passive, sometimes legacies, he focuses on what are the territorial factors, endogenous and exogenous, that can explain his current evolutions, particularly from the point of view of the residential economy versus productive economy. Lastly, in support of several territorial transformation projects (industrial and urban wastelands, in particular) carried out or in the process of being completed within the Val de Fensch Urban Community, it shows the importance of engaging still industrial territories in a futuristic and anticipatory approach.

In diesem Artikel, befragt sich der Autor nach den Kapazitäten eines Industriegebiets in voller Strukturwandel, das sich im Norden des alten Lothringens in der Nähe der luxemburgischen Grenze befindet, Sich neu zu erfinden und die Wege seiner Widerstandsfähigkeit zu finden. Nachdem er Erbschaften beschrieben hat, manchmal passiv oder legs, untersucht er die territorialen endogen und exogen Faktoren, die seine aktuellen Entwicklungen erklären können, insbesondere aus der Sicht der Wohnwirtschaft versus der produktiven Wirtschaft. Schließlich zeigt er zur Unterstützung mehrerer durchgeführter oder in Vorbereitung befindlicher Projekte zur territorialen Umgestaltung innerhalb der Verbandsgemeinde Val de Fensch, wie wichtig es ist, die noch bestehenden Industriegebiete in einen vorausschauenden und vorausschauenden Ansatz einzubinden.

\section{INDEX}

Keywords : Territorial resilience, industrial inheritances, urban transformation, productive and residential economy, local development, territorial recomposition.

Schlüsselwörter : Territoriale Widerstandskraft, industrielles Erbe, städtischer Wandel, Produktive und Wohnwirtschaft, lokale Entwicklung, territoriale Neuordnung.

Mots-clés : Résilience territoriale, héritages industriels, transformation urbaine, économie productive et résidentielle, développement local, recomposition territoriale. 


\section{AUTEUR}

\section{ERIC MAROCHINI}

Docteur en géographie/aménagement - Chercheur associé au sein du laboratoire LOTERR -

Université de Lorraine. Directeur Général des Services - Communauté de Communes du Saulnois

- 14 Ter Place de la Saline - 57170 Château-Salins - eric.marochini@cc-saulnois.fr 